\title{
NEW ENERGETIC SELECTION PRINCIPLE IN DIFFERENTIAL EVOLUTION
}

\author{
Vitaliy Feoktistov \\ Centre de Recherche LGI2P, Site EERIE, EMA \\ Parc Scientifique Georges Besse, 30035 Nimes, France \\ Vitaliy.Feoktistov@ema.fr \\ Stefan Janaqi \\ Centre de Recherche LGI2P, Site EERIE, EMA \\ Parc Scientifique Georges Besse, 30035 Nimes, France \\ Stefan.Janaqi@ema.fr
}

\begin{abstract}
Key words: differential evolution, evolutionary algorithms, heuristics, optimization, selection.
Abstract: The Differential Evolution (DE) algorithm goes back to the class of Evolutionary Algorithms and inherits its philosophy and concept. Possessing only three control parameters (size of population, differentiation and recombination constants) DE has promising characteristics of robustness and convergence. In this paper we introduce a new principle of Energetic Selection. It consists in both decreasing the population size and the computation efforts according to an energetic barrier function which depends on the number of generation. The value of this function acts as an energetic filter, through which can pass only individuals with lower fitness. Furthermore, this approach allows us to initialize a population of a sufficient (large) size. This method leads us to an improvement of algorithm convergence.
\end{abstract}

\section{INTRODUCTION}

Evolutionary Algorithms increasingly become the primary method of choice for optimization problems that are too complex to be solved by deterministic techniques. They are universal, robust, easy to use and inherently parallel. The huge number of applications and continuous interest prove it during several decades (Heitkötter and Beasley, 2000; Beasley, 1997). In comparison with the deterministic methods Evolutionary Algorithm require superficial knowledge about the problem being solved. Generally, it is only necessary that the algorithm is able to evaluate the objective function for a given set of input parameters. Nevertheless, in most cases such heuristics take less time to find the optimum than, for example, gradient methods. One of the latest breakthroughs in the evolutionary computation is the Differential Evolution algorithm.

\section{DIFFERENTIAL EVOLUTION}

Differential Evolution is a recently invented global optimization technique (Storn and Price, 1995). It can be classified as an iterative stochastic method. Enlarging the Evolutionary Algorithms' group, DE turns out to be one of the best population-based optimizers
(Storn and Price, 1996). In the following lines we give a brief description of DE algorithm.

An optimization problem is represented by a set of variables. Let these variables form a $D$-dimensional vector in continuous space $X=\left(x_{1}, \ldots, x_{D}\right) \in \mathbb{R}^{D}$. Let there be some criterion of optimization $f(X)$ : $\mathbb{R}^{D} \rightarrow \mathbb{R}$, usually named either fitness or cost function. Then the goal of optimization is to find the values of the variables that minimize the criterion, i.e. to find

$$
X^{*}: f\left(X^{*}\right)=\min _{X} f(X)
$$

Often, the variables satisfy boundary constraints

$$
L \leq X \leq H: \quad L, H \in \mathbb{R}^{D}
$$

As all Evolutionary Algorithms, DE deals with a population of solutions. The population $\mathbb{P}$ of a generation $g$ has $N P$ vectors, so-called individuals of population. Each such individual represents a potential optimal solution.

$$
\mathbb{P}^{g}=X_{i}^{g}, \quad i=1, \ldots, N P
$$

In turn, the individual contains $D$ variables, so called genes.

$$
X_{i}^{g}=x_{i, j}^{g}, \quad j=1, \ldots, D
$$

The population is initialized by randomly generating individuals within the boundary constraints,

$$
\mathbb{P}^{0}=x_{i, j}^{0}=\operatorname{rand}_{i, j} \cdot\left(h_{j}-l_{j}\right)+l_{j}
$$


where rand function generates values uniformly in the interval $[0,1]$.

Then, for each generation the individuals of a population are updated by means of a reproduction scheme. Thereto for each individual ind a set of other individuals $\pi$ is randomly extracted from a population. To produce a new one the operations of Differentiation and Recombination are applied one after another. Next, the Selection is used to choose the best. Now briefly consider these operations.

Here, we show a typical model of the Differentiation, others can be found in (Storn and Price, 1995). For that, three different individuals $\pi=\left\{\xi_{1}, \xi_{2}, \xi_{3}\right\}$ are randomly extracted from a population. So, the result, a trial individual, is

$$
\tau=\xi_{3}+F \cdot\left(\xi_{2}-\xi_{1}\right),
$$

where $F>0$ is the constant of differentiation.

After, the trial individual $\tau$ is recombined with updated one ind. The Recombination represents a typical case of a genes' exchange. The trial one inherits genes with some probability. Thus,

$$
\omega_{j}= \begin{cases}\tau_{j} & \text { if } \operatorname{rand}_{j}<C r \\ i n d_{j} & \text { otherwise }\end{cases}
$$

where $j=1, \ldots, D$ and $C r \in[0,1)$ is the constant of recombination.

The Selection is realized by comparing the cost function values of updated and trial individuals. If the trial individual better minimizes the cost function, then it replaces the updated one.

$$
\text { ind }= \begin{cases}\omega & \text { if } f(\omega) \leq f(i n d) \\ \text { ind } & \text { otherwise }\end{cases}
$$

Notice that there are only three control parameters in this algorithm. These are $N P$ - population size, $F$ and $\mathrm{Cr}$ - constants of differentiation and recombination accordingly. As for the terminal conditions, one can either fix the number of generations $g_{\max }$ or a desirable precision of a solution VTR (value to reach).

The pattern of DE algorithm is presented in the following way (see algorithm 1).

\section{ENERGETIC APPROACH}

We introduce a new energetic approach which can be applied to population-based optimization algorithms including DE. This approach may be associated with the processes taking place in physics.

Let there exist a population $\mathbb{P}$ consisting of $N P$ individuals. Let us define the potential of individual as its cost function value $\varphi=f($ ind $)$. Such potential shows the remoteness from the optimal solution $\varphi^{*}=f\left(i n d^{*}\right)$, i.e. some energetic distance (potential) that should be overcome to reach the optimum.

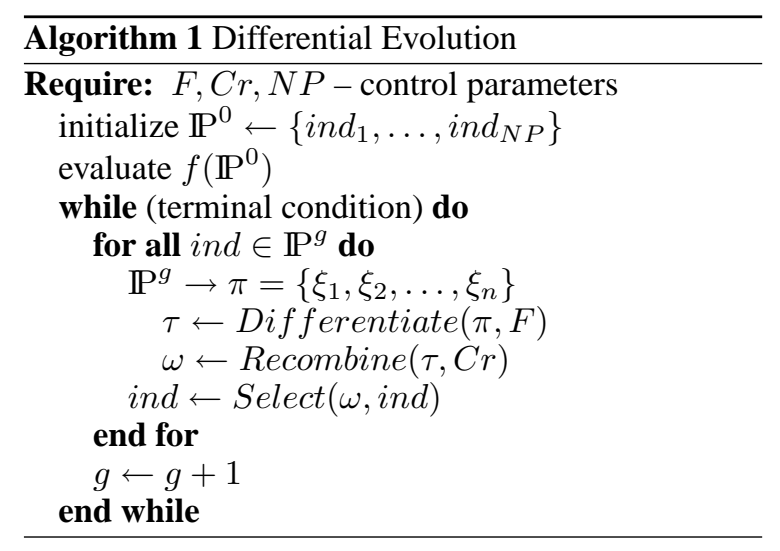

Then, the population can be characterized by superior and inferior potentials $\varphi_{\max }=\max f\left(\right.$ ind $\left._{i}\right)$ and $\varphi_{\min }=\min f\left(\right.$ ind $\left._{i}\right)$. As the population evaluates the individuals take more optimal energetic positions, the closest possible to the optimum level. So if $t \rightarrow \infty$ then $\varphi_{\max }(t) \rightarrow \varphi_{\min }(t) \rightarrow \varphi^{*}$, where $t$ is an elementary step of evolution. Approaching the optimum, apart from stagnation cases (Lampinen and Zelinka, $2000)$, can be as well expressed by $\varphi_{\max } \rightarrow \varphi_{\min }$ or $\left(\varphi_{\max }-\varphi_{\min }\right) \rightarrow 0$. By introducing the potential difference of population $\triangle \varphi(t)=\varphi_{\max }(t)-\varphi_{\min }(t)$ the condition of optimality is represented as

$$
\triangle \varphi(t) \rightarrow 0
$$

In other words, the optimum is achieved when the potential difference is closed to 0 or to some desired precision $\varepsilon$. The value $\triangle \varphi(t)$ is proportional to the algorithmic efforts, which are necessary to find the optimal solution.

Thus, the action $A$ done by the algorithm in order to pass from one state $t_{1}$ to another $t_{2}$ is

$$
A\left(t_{1}, t_{2}\right)=\int_{t_{1}}^{t_{2}} \triangle \varphi(t) d t
$$

We introduce then the potential energy of population $E_{p}$ that describes total computational expenses.

$$
E_{p}=\int_{0}^{\infty} \triangle \varphi(t) d t
$$

Notice that the equation (11) graphically represents the area $S_{p}$ between two functions $\varphi_{\max }(t)$ and $\varphi_{\min }(t)$.

Let us remind that our purpose is to increase the algorithm convergence. Logically, the convergence is proportional to computational efforts. It is obvious the less is potential energy $E_{p}$ the less computational efforts are needed. Thus, by decreasing the potential energy $E_{p} \equiv S_{p}$ we augment the convergence rate of the algorithm. Hence, the convergence increasing 


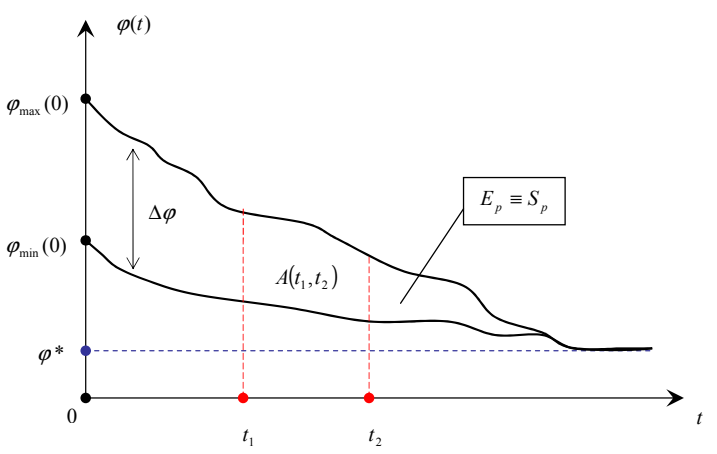

FIG. 1 - Energetic approach.

aim transforms into a problem of potential energy minimization (or $S_{p}$ minimization).

$$
E_{p}^{*}=\min _{\Delta \varphi(t)} E_{p}(\triangle \varphi(t))
$$

\section{NEW ENERGETIC SELECTION PRINCIPLE}

\subsection{Idea}

We apply the above introduced Energetic Approach to the DE algorithm. As an elementary evolution step $t$ we choose a generation $g$.

In order to increase the convergence rate we minimize the potential energy of population $E_{p}$ (fig.1). For that a supplementary procedure is introduced at the end of each generation $g$. The main idea is to replace the superior potential $\varphi_{\max }(g)$ by so called energetic barrier function $\beta(g)$. Such function artificially plays down the potential difference of generation $\triangle \varphi(g)$.

$$
\beta(g) \leq \varphi_{\max }(g), \quad \forall g \in\left[1, g_{\max }\right]
$$

From an algorithmic point of view this function $\beta(g)$ serves as an energetic filter for the individuals passing into the next generation. Thus, only the individuals with potentials less than the current energetic barrier value can participate in the next evolutionary cycle (fig.2).

Practically, it leads to the decrease of the population size $N P$ by rejecting individuals which violate the condition (14).

$$
f(\text { ind }) \leq \beta(g)
$$

\subsection{Energetic Barriers}

Here, we show some examples of the energetic barrier function. At the beginning we outline the variables which this function should depend on. Firstly,

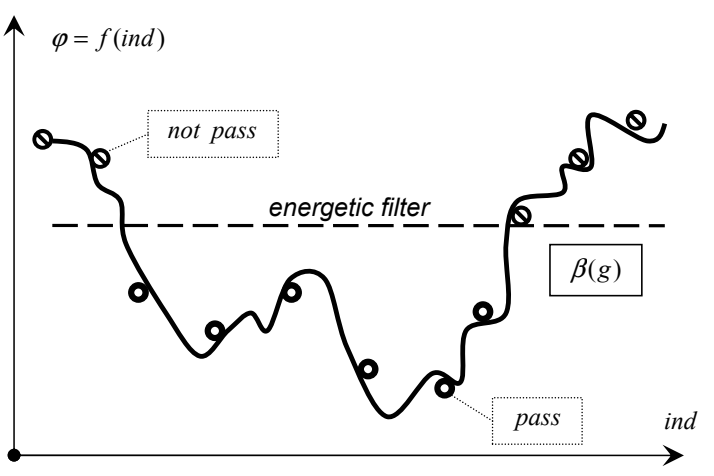

FIG. 2 - Energetic filter.

this is the generation variable $g$, which provides a passage from one evolutionary cycle to the other. Secondly, it should be the superior potential $\varphi_{\max }(g)$ that presents the upper bound of the barrier function. And thirdly, it should be the inferior potential $\varphi_{\min }(g)$ ensuring the low bound of the barrier function (fig.3).

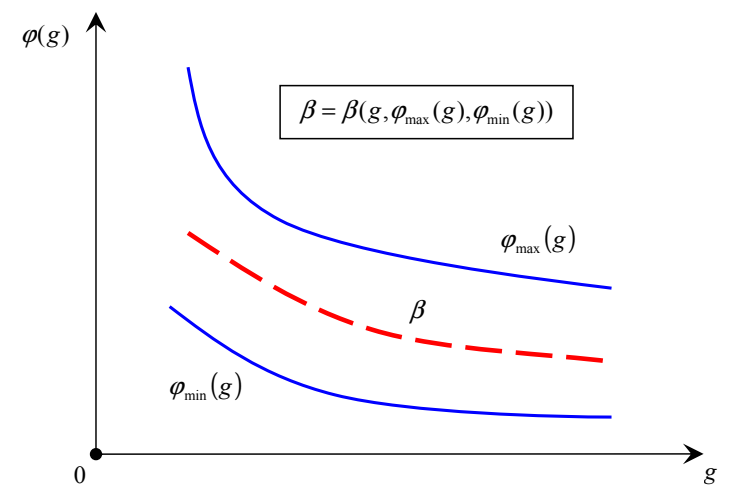

FIG. 3 - Energetic barrier function.

Linear energetic barriers. The simplest example is the use of a proportional function. It is easy to obtain by multiplying either $\varphi_{\min }(g)$ or $\varphi_{\max }(g)$ on a constant $K$.

In the first case, the value $\varphi_{\min }(g)$ is always stored in the program as the current best value of the cost function. So, the energetic barrier looks like

$$
\beta_{1}(g)=K \cdot \varphi_{\min }(g), \quad K>1
$$

The constant $K$ is selected to satisfy the energetic barrier condition (13).

In the second case, firstly a little procedure is necessary to find superior potential (maximal cost function value of the population) $\varphi_{\max }(g)$. Here, the energetic barrier is

$$
\beta_{2}(g)=K \cdot \varphi_{\max }(g), \quad K<1
$$


$K$ should not be too small in order to provide a smooth decrease of the population size $N P$.

An advanced example would be a superposition of the potentials.

$$
\beta_{3}(g)=K \cdot \varphi_{\min }(g)+(1-K) \cdot \varphi_{\max }(g)
$$

So, with $K<1$ the energetic barrier function is always found between the potential functions. Now, by adjusting $K$ it is easier to get the smoothed reduction of the population without condition violation (13). Examples of the energetic barrier functions are shown on the figure (fig.4).

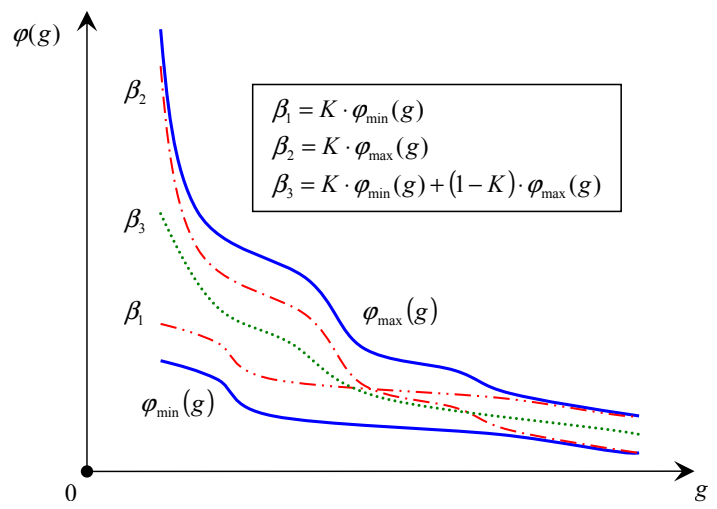

FIG. 4 - Linear energetic barriers.

Nonlinear energetic barriers. As we can see the main difficulty of using the linear barriers appears when we try to define correctly the barrier function in order to provide a desired dynamics of the population reduction. Taking into consideration that $\varphi_{\max } \rightarrow$ $\varphi_{\min }$ when the algorithm converges, the ideal choice for the barrier function is a function which begins at a certain value between $\varphi_{\min }(0)$ and $\varphi_{\max }(0)$ and converges to $\varphi_{\max }\left(g_{\max }\right)$.

Thereto, we propose an exponential function $K(g)$

$$
K(g)=K_{l}+\left(K_{h}-K_{l}\right) \cdot e^{\left(-\frac{T}{g_{\max }} \cdot g\right)}
$$

This function smoothly converges from $K_{h}$ to $K_{l}$. The constant $T$, so called temperature, controls the convergence rate. The functional dependence on the temperature constant $K(T)$ is represented on the figure (fig.5).

By substituting the constant $K$ in the equations (1517) for the exponential function (18) we can supply the energetic barrier function with improved turning (fig.6).

\subsection{Advantages}

Firstly, such principle of energetic selection permits to initialize the population of a sufficiently large

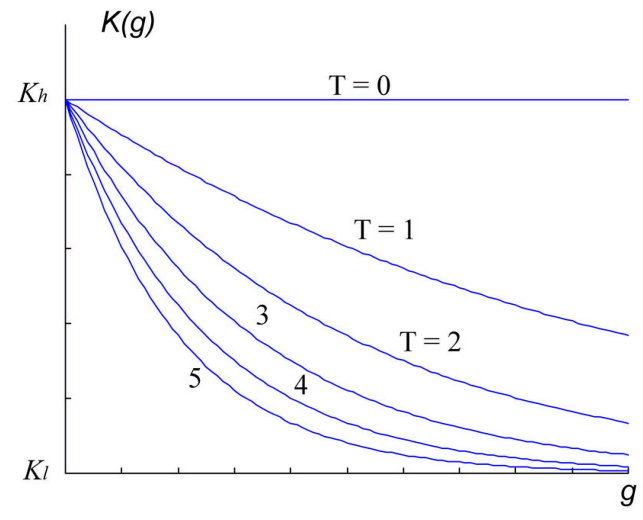

FIG. 5 - Exponential function $K(g, T)$.

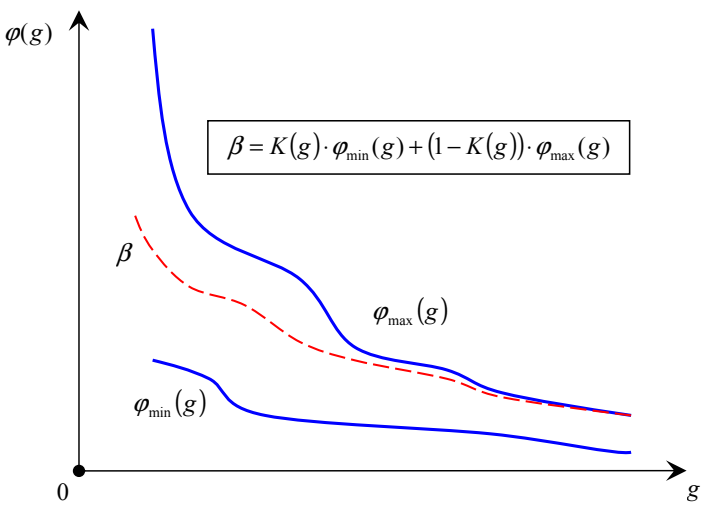

FIG. 6 - Nonlinear energetic barrier.

size. This fact leads to better (careful) exploration of a search space on the initial generations as well as it augments the probability of finding the global optimum.

Secondly, the introduction of the energetic barrier function decreases the potential energy of the population and thereby increases the algorithm convergence.

Thirdly, a double selection principle is applied. The first one is a usual DE selection for each individual of a population. Here, there is no reduction of the population size. And the second one is a selection of the best individuals which pass in the next generation, according to the energetic barrier function. It leads to the reduction of the population size.

Remark. Notice that a considerable reduction of the population size occurs at the beginning of the evolutionary process. For more efficient exploitation of this fact a population should be initialized with greatly larger size $N P_{0}$ than usually. Then, when the population shrinks to a certain size $N P_{f}$, it is necessary to stop the energetic selection procedure. This forced stopping is explained by possible stagnation 
and not enough efficient search in a small size population (Lampinen and Zelinka, 2000; Price, 2003).

\section{COMPARISON OF RESULTS}

In order to test our approach we chose three test functions (19) from a standard test suite for Evolutionary Algorithms (Whitley et al., 1996). The first two functions, Sphere $f_{1}$ and Rosenbrock's function $f_{2}$, are classical De Jong testbads (Jong, 1975). Sphere is a "dream" of every optimization algorithm. It is smooth, unimodal and symmetric function. The performance on the Sphere function is a measure of the general efficiency of the algorithm. Whereas the Rosenbrock's function is a nightmare. It has a very narrow ridge. The tip of the ridge is very sharp and it runs around a parabola. The third function, Rotated Ellipsoid $f_{3}$, is a true quadratic non separable optimization problem.

$$
\begin{aligned}
& f_{1}(X)=\sum_{i=1}^{3} x_{i}^{2} \\
& f_{2}(X)=100\left(x_{1}^{2}-x_{2}\right)^{2}+\left(1-x_{1}\right)^{2} \\
& f_{3}(X)=\sum_{i=1}^{20}\left(\sum_{j=1}^{i} x_{j}\right)^{2}
\end{aligned}
$$

We fixed the differentiation $F$ and recombination $\mathrm{Cr}$ constants the same for all functions. $F=0.5$. Recombination $\mathrm{Cr}=0$ (there is no recombination) in order to make the DE algorithm rotationally invariant (Salomon, 1996; Price, 2003). The terminal condition of algorithm is a desirable precision of optimal solution $V T R$ (value to reach). It is fixed for all tests as $V T R=1.0 e-6$. We count the number of function evaluations NFE needed to reach the VTR. The initial data are shown in the table 1.

\begin{tabular}{cccccc}
\hline \hline$f_{i}$ & $D$ & $N P$ & $N P_{0}$ & $N P_{f}$ & $K$ \\
\hline 1 & 3 & 30 & 90 & 25 & 0.50 \\
2 & 2 & 40 & 120 & 28 & 0.75 \\
3 & 20 & 200 & 600 & 176 & 0.15 \\
\hline \hline
\end{tabular}

TAB. 1 - Initial test data.

For DE with energetic selection principle the initial population size was chosen three times larger than in the classical DE scheme : $N P_{0}=3 \cdot N P$. The forced stopping was applied if the current population became smaller than $N P$. Hence $N P_{f} \leq N P$. As an energetic barrier function the linear barrier $\beta_{3}(g)$ was selected (17). So, $K$ is an adjusting parameter for barrier turning. $D$ is the dimension of test functions.
The average results of 10 runs for both the classical DE scheme and DE with energetic selection principle are summarized in the table 2 .

\begin{tabular}{rrrr}
\hline \hline$f_{i}$ & $N F E_{c l}$ & $N F E_{e s}$ & $\delta, \%$ \\
\hline 1 & 1088.7 & 912.4 & 16,19 \\
2 & 1072.9 & 915.3 & 14,69 \\
3 & 106459.8 & 94955.6 & 10,81 \\
\hline \hline
\end{tabular}

TAB. 2 - Comparison the classical DE scheme $(c l)$ and DE with energetic selection principle (es).

The numbers of function evaluations ( $N F E$ 's) were compared. It is considered that $N F E_{c l}$ value is equal to $100 \%$ therefore the relative convergence amelioration in percentage wise can be defined as

$$
\delta=1-\frac{N F E_{e s}}{N F E_{c l}}
$$

Thus, $\delta$ may be interpreted as the algorithm improvement.

\section{CONCLUSION}

The variation of the population size of populationbased search procedures presents a rather promising trend. In this article we have examined its decrease. The proposed energetic approach explains a theoretical aspect of such population reduction. The efficiency of the new energetic selection principle based on this energetic approach is illustrated by the example of the DE algorithm. The given innovation provides more careful exploration of a search space and leads to the convergence improvement. Thus, the probability of the global optimum finding is increased. Further works are carried on the methods of increasing the population size.

\section{REFERENCES}

Beasley, D. (1997). Possible applications of evolutionary computation. In Bäck, T., Fogel, D. B., and Michalewicz, Z., editors, Handbook of Evolutionary Computation, pages A1.2 :1-10. IOP Publishing Ltd. and Oxford University Press, Bristol, New York.

Heitkötter, J. and Beasley, D. (2000). Hitch Hiker's Guide to Evolutionary Computation : A List of Frequently Asked Questions (FAQ).

Jong, K. A. D. (1975). An analysis of the behavior of a class of genetic adaptive systems. $\mathrm{PhD}$ thesis, University of Michigan. 
Lampinen, J. and Zelinka, I. (7-9 June 2000). On stagnation of the differential evolution algorithm. In Osmera, P., editor, Proceedings of MENDEL'2000, 6-th International Mendel Conference on Soft Computing, pages 76-83, Brno University of Technology, Faculty of Mechanical Engineering, Institute of Automation and Computer Science, Brno (Czech Republic).

Price, K. (2003). New Ideas in Optimization, Part 2 : Differential Evolution. McGraw-Hill, London, UK.

Salomon, R. (1996). Re-evaluating genetic algorithm performance under coordinate rotation of benchmark functions : A survey of some theoretical and practical aspects of genetic algorithms. BioSystems, 39 :263278.

Storn, R. and Price, K. (1995). Differential evolution - a simple and efficient adaptive scheme for global optimization over continuous spaces. Technical Report TR-95-012, International Computer Science Institute, Berkeley, CA.

Storn, R. and Price, K. (1996). Minimizing the real functions of the ICEC'96 contest by differential evolution. In IEEE International Conference on Evolutionary Computation, pages 842-844, Nagoya. IEEE, New York, NY, USA.

Whitley, D., Rana, S. B., Dzubera, J., and Mathias, K. E. (1996). Evaluating evolutionary algorithms. Artificial Intelligence, 85(1-2) :245-276. 